\title{
Tendency of Discreteness of the Solar Amplitude and Intercycle Relatedness
}

\author{
Akio Yoshida ${ }^{1}$ and Ryan Sayre ${ }^{2}$ \\ ${ }^{1}$ Center for Integrated Research and Education of Natural Hazards, Shizuoka University, 836 Ohya, Suruga-ku, \\ Shizuoka 422-8529, Japan \\ ${ }^{2}$ Department of Anthropology, Yale University, P.O. Box 208105, New Haven, CT 06520, USA
}

Correspondence should be addressed to Akio Yoshida, akio.yoshi@nifty.com

Received 13 December 2011; Revised 21 January 2012; Accepted 17 February 2012

Academic Editor: J. P. Rozelot

Copyright ( 2012 A. Yoshida and R. Sayre. This is an open access article distributed under the Creative Commons Attribution License, which permits unrestricted use, distribution, and reproduction in any medium, provided the original work is properly cited.

\begin{abstract}
We suggest that the average of the sunspot number (SSN) over a cycle offers a more appropriate index than the maximum value of the SSN in the evaluation of the activity of solar cycles. We then show that the average SSN has a tendency to take discrete values, that is, integral multiples of 20. This remarkable finding is supported by the fact that SSNs around the maximum are likely to take some particular values. Further, it is shown that there exists a positive correlation in the amplitude between even-numbered cycles and succeeding odd-numbered cycles and an inverse correlation between $(2 n+1)$ cycles and $(2 n+4)$ cycles where $n$ represents an integer. If these two correlations are combined, it turns out that there exist two mutually independent series of cycles which do not mix or merge.
\end{abstract}

\section{Introduction}

The solar cycle, that is, the waxing and waning of solar activity over periods of roughly 11 years, is usually measured by increases and decreases in the sunspot number (SSN). By convention, the maximum SSN has been taken as representative of a given cycle's amplitude. The minimum, in turn, has been taken to serve as a cycle's starting and ending points. Here we show that the average SSN over a cycle is well correlated with the maximum SSN in the cycle and propose that the former quantity offers a more appropriate index in the evaluation of the activity of solar cycles.

In a previous study we showed that the correlation between the monthly smoothed SSN and the maximum SSN of the succeeding cycle is highest for the SSN at a point three years before the minimum [1] In this paper, it is shown that this proves also to be the case for the monthly smoothed SSN and the average SSN. That is, the correlation coefficient between the average SSN over a cycle and the smoothed SSN at a dividing point becomes largest when cycles are cut at a point three years before the minimum. The correlation between the average SSN and the maximum SSN is also highest when cycles are divided at a point three years before the minimum. These findings suggest that the SSN in the final several years of a cycle may include some critical information about the amplitude of the following cycle. We consider this problem in Section 4 where some more relevant observational facts are presented.

The main aim of this paper is to introduce two intriguing and somewhat surprising behaviors made visible when one takes the average SSN as the amplitude of the solar cycle. We first show that the amplitude tends to take discrete values. We then go on to discuss an interrelatedness of cycles which we believe to have potential implications for the long-range predictability of solar activity.

\section{Discreteness of the Amplitude of Solar Cycles}

The idea that the average SSN over a cycle may be more appropriate than the maximum SSN in the evaluation of the activity of solar cycles occurred to us when we observed a diagram depicting monthly smoothed sunspot numbers 


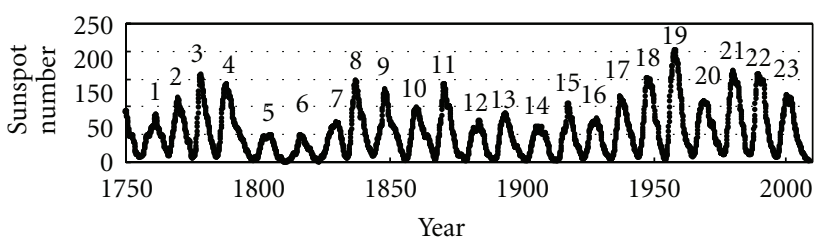

FIGURE 1: Monthly smoothed sunspot number since 1750. Numerals in the map indicate cycle number.

since 1750, which showed significant difference in the pattern of change around the maximum, with steep peaks in some cycles, and more rounded peaks in others (Figure 1). Data related to the monthly smoothed SSN was obtained from the Solar Influence Data Analysis Center (http://www.sidc.be/ sunspot-data/). In this paper the maximum or the minimum SSN means the maximum or minimum monthly smoothed SSN, and in cases in which the same value succeeds twice or more, the last one is taken to specify the point where the solar activity becomes a maximum or a minimum.

Figure 2(a) shows scatter plots between the maximum SSN and the average SSN over individual solar cycles when the cycles are divided at the minimum. Figure 2(b) shows that when the cycles are divided at a point three years before the minimum. Note that the correlation coefficient between the maximum SSN and the average SSN is larger in the latter case. In fact, the correlation coefficient when solar cycles are divided at the minimum and at points one, two, three, four, and five years before the minimum is $0.964(0.916,0.984)$, $0.966(0.921,0.985), 0.970(0.929,0.987), 0.972(0.934$, $0.988), 0.968(0.923,0.986)$, and $0.940(0.862,0.975)$, respectively, where numerals in parentheses indicate lower and upper limits of the $95 \%$ confidence interval of the correlation coefficient. Consequently, the largest correlation coefficient is obtained when solar cycles are divided at a point three years before the minimum, though those values do not differ much from each other. Figure 3 shows similar correlation between the sum of the SSN over a cycle and the maximum SSN. The correlation coefficient when cycles are divided at the minimum (Figure 3(a)) is $0.935(0.851,0.972)$ and the correlation coefficient when cycles are divided at a point three years before the minimum (Figure 3(b)) is $0.919(0.815,0.965)$, respectively. Numerals in parentheses indicate lower and upper limits of the $95 \%$ confidence interval of the correlation coefficient. It is interesting that the correlation when cycles are divided at the minimum is better than the correlation when cycles are divided at a point three years before the minimum in this case. This notwithstanding, for every case of the dividing point, the correlation coefficient between the maximum SSN and the sum of the SSN is smaller compared to that between the maximum SSN and the average SSN. This result demonstrates that the average SSN rather than the sum of the SSN is a more appropriate quantity for representing the amplitude of the solar cycle.

Similar to the correlation between the average SSN and the maximum SSN, the best correlation between the average SSN and the SSN at the dividing point is obtained when solar cycles are divided at a point three years before the minimum.

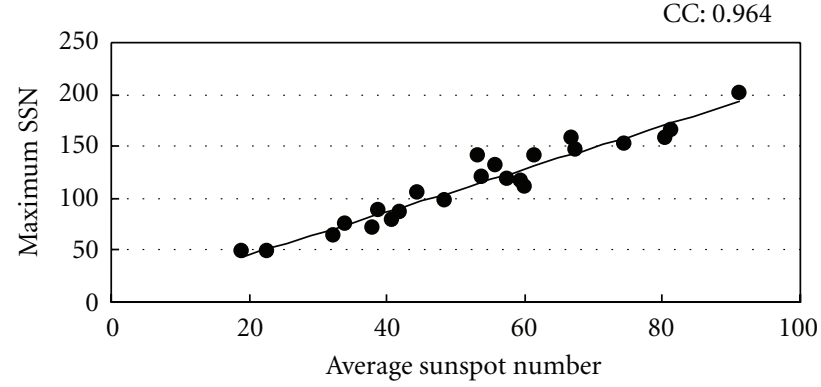

(a)

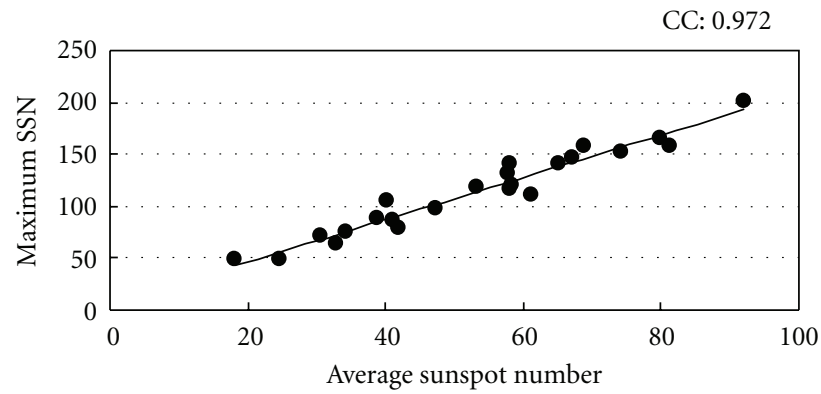

(b)

FIgURE 2: (a) Peak sunspot number versus average sunspot number when solar cycle is divided at the minimum. (b) is the same as (a); however the dividing point is set at three years before the minimum. CC on the top right-hand side of each map represents the correlation coefficient.

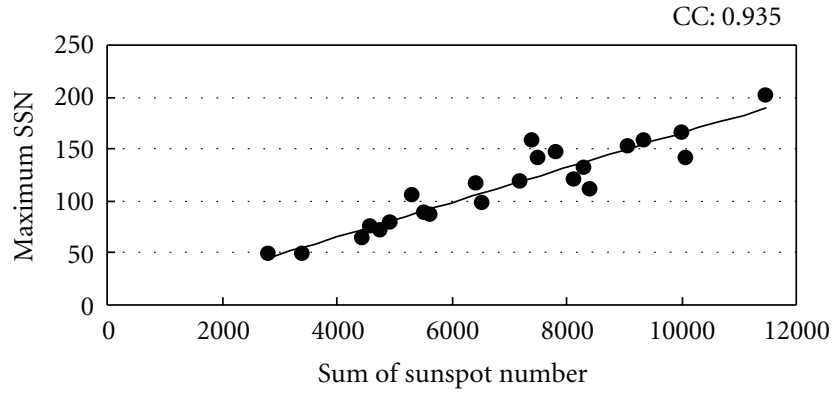

(a)

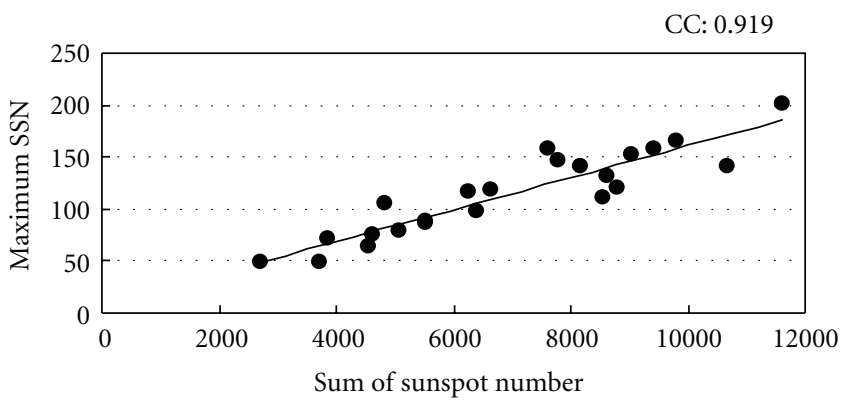

(b)

Figure 3: (a) and (b) are the same as Figures 2(a) and 2(b) except that sum of sunspot number over a cycle is taken instead of the average sunspot number. 
Figure 4(a) shows scatter plots between the minimum SSN and the average SSN over individual solar cycles when the cycles are divided at the minimum. Figure 4(b) shows that between the SSN at a point three years before the minimum and the average SSN when the cycles are divided at the point. The correlation coefficient between the minimum SSN and the average SSN divided at the minimum is 0.783 (0540, 0.906), while that between the SSN at a point three years before the minimum and the average SSN over a cycle divided at the same point is $0.890(0.749,0.954)$, where numerals in the parentheses show lower and upper limits of the $95 \%$ confidence interval of the correlation coefficient. The correlation coefficient when the dividing point is taken at a point one year, two years, three years, four years, and five years before the minimum is $0.706(0.414,0.866), 0.752$ (0.493, 0.889), $0.890(0.749,0.954), 0.871$ (0.715, 0.944), and $0.829(0.632,0.925)$, respectively. Note that the best correlation is again obtained when solar cycles are divided at a point three years before the minimum and that the value of the correlation coefficient is nearly equal to the upper limit of the 95\% interval of the correlation coefficient obtained when cycles are divided at the minimum, suggesting that a point three years before the minimum is more appropriate than the minimum as the cycle's ending and starting points. Here, we would like to add that, for each of the cases, the previously given correlation coefficient between the SSN at the dividing point and the average SSN is larger than the correlation coefficient that was obtained between the SSN at the dividing point and the maximum SSN in the following cycle [1].

We take all of these results to suggest that the average SSN over the course of a cycle is a proper quantity for representing the amplitude of a cycle and the point three years prior to the minimum may be the most appropriate point at which we define a cycle beginning/ending point. Although the latter maintenance does not accord with the conventional idea, we think that something meaningful should exist in the previous results where the largest correlation coefficient is obtained when a cycle is divided at a point three years before the minimum. We consider their implications in Section 4.

Taking the average SSN as representative of the amplitude of a solar cycle, we now show that a number of intriguing phenomena become visible. We use in the following analysis the average SSN obtained by dividing cycles at a point three years before the minimum. However, the overall results do not change if we use the average SSN calculated by taking the dividing point at the minimum.

Figure 5(a) illustrates the cumulative number of cycles in order of the amplitude over the 23 cycles since 1750 . One can note a tendency of the average SSN to distribute around the values of 40 and 60, that is, multiples of 20 from the map. This tendency is clearly visible when the cumulative number of cycles is plotted in order of the difference of the average SSN from the nearest multiple of 20 (Figure 5(b)). Note that the clustering of the plotted points is hardly seen when the maximum SSN is taken as the representative of the amplitude (Figure 5(c)). We can see in Figure 5(b) that 12 of the total 23 points fall in the range of -2.5 to 2.5 (i.e., one fourth of the potential range). If it is assumed that the amplitude of solar

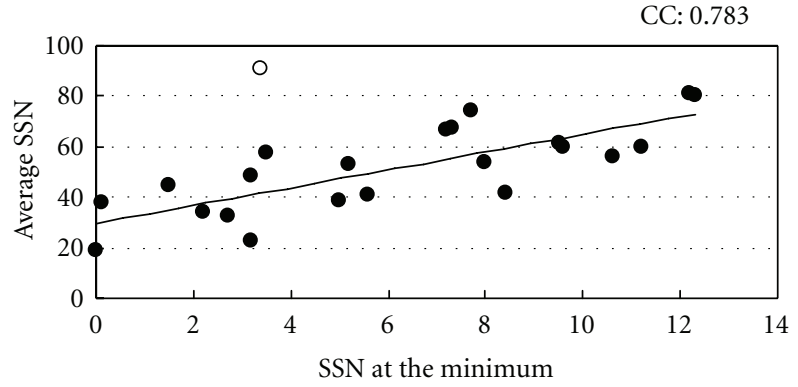

(a)

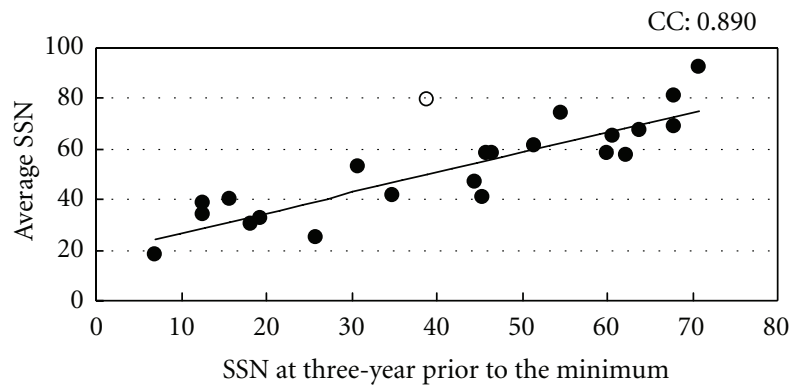

(b)

Figure 4: (a) Average sunspot number divided at the minimum versus the minimum sunspot number. (b) Average sunspot number divided at a point three years before the minimum versus the sunspot number at the dividing point. CC on the top right-hand side of each map represents the correlation coefficient. Outliers plotted by white circles in the maps are omitted in the calculation of the correlation coefficient.

activity can take a random continuous value, the statistical probability of this occurring is calculated to be as small as 4.6 $\times 10^{-3}$. This probability is so small that the hypothesis that the amplitude takes a continuous value seems to be denied assuredly. However, it is to be noted that the deduction is dependent on the presupposition itself. If the assumption is not correct, that is, in case the SSNs are liable to take some particular value and its multiples, the conclusion would become a different one. We show next, however, that that is most improbable.

Figure 6 shows the number of months that enter in each bin of SSN with an interval of 5, separating the cycle into three periods, around the maximum (two years before each maximum to two years after the maximum), the rising phase, and the decaying phase. Note the intriguing distribution of the number in Figure 6(a). The SSN at the period of the solar maximum swarms around several particular values, centered at the bin of $60-65,105-110$, and 145-150. No doubt this tendency should afford the basis for our finding that the average SSN takes the value around multiples of 20. It is to be noted that such a feature of swarming is not so clear for the SSN in other periods, that is, in the rising and decaying phases (Figures 6(b) and 6(c)), though small bumps are observed around the bin of 45-50 for the rising phase and around the bins of 25-30 and 65-70 for the decaying phase. For these phases, the fact that the ratio of the SSN takes the value $5-10$ or $10-15$ is notable. We think 


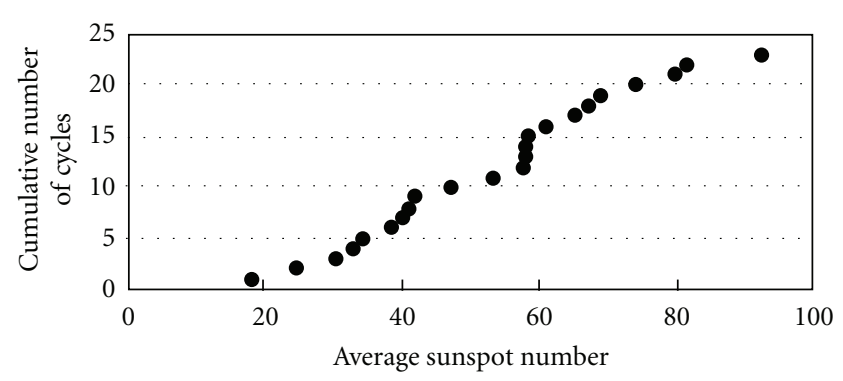

(a)

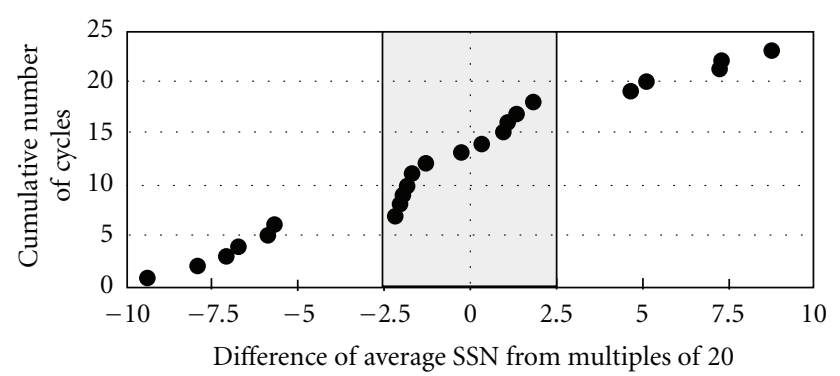

(b)

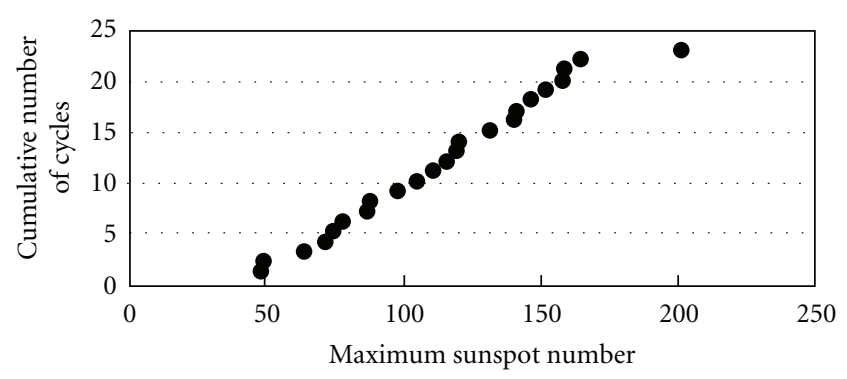

(c)

FIgUre 5: (a) Cumulative number of cycles in order of the average sunspot number over a cycle. (b) Cumulative number of cycles in order of the difference of the average sunspot number from the nearest multiple of 20. (c) Cumulative number of cycles in order of the maximum sunspot number. In Figures 5, 7, 8, and 9 the average sunspot number is obtained by dividing the solar cycle three years before the minimum.

that the aforementioned facts, especially the bumps in the distribution seen in Figure 6(a), assure us that the tendency of discreteness of solar amplitude does not stem from an artificial cause such as the procedure to calculate the monthly smoothed SSN, but is characteristic of the solar activity at its maximum period.

\section{Intercycle Relatedness of the Amplitude}

The scatter plots in Figures 7(a) and 7(b) show the relation between the average SSN of odd-numbered solar cycles and that of their preceding even-numbered cycles (i.e., between the average SSN for $(2 n+1)$ cycles and that for $2 n$ cycles), and the relation between the average SSN of odd-numbered cycles and that of their succeeding even-numbered cycles, (i.e., between the average SSN for $(2 n+1)$ cycles and that for $(2 n+2)$ cycles), respectively. A positive correlation is

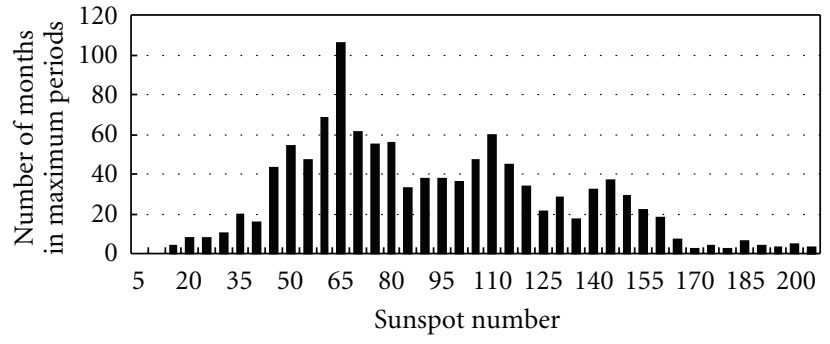

(a)

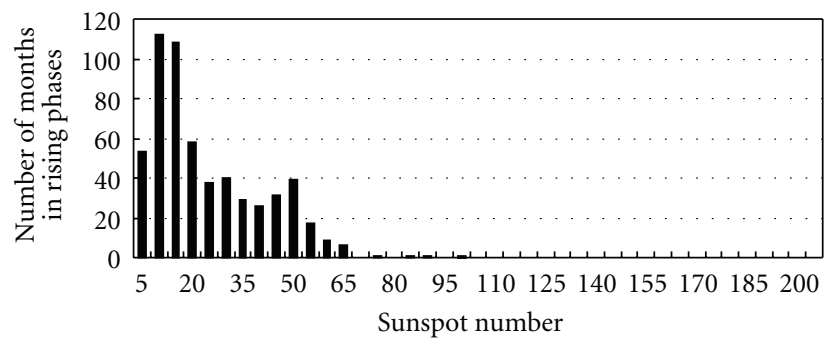

(b)

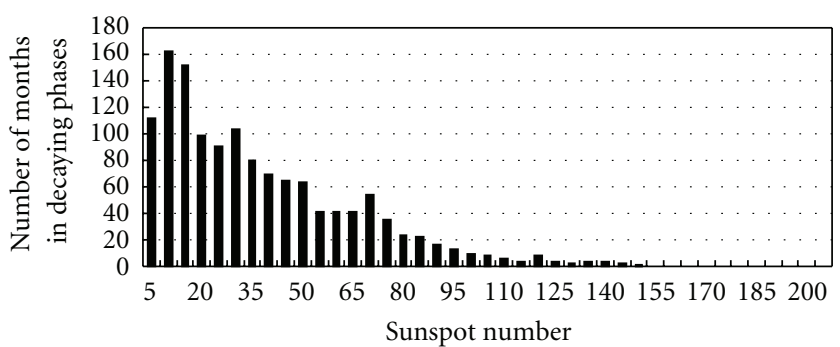

(c)

FIGURE 6: Frequency distribution of monthly smoothed SSN during (a) periods at solar maximum (the period from two years before each of the maximums of the SSN to two years after the maximum), (b) periods of rising phase (the period from the minimum SSN to two years before the beginning of the solar maximum period), and (c) periods of decaying phase (the period from the end of each of the solar maximum periods to the minimum SSN), respectively. The abscissa and the ordinate represent the monthly SSN and number of months, respectively.

observed for both cases, but the correlation in the former case is much better, carrying correlation coefficient of 0.985 (lower and upper limits of the $95 \%$ confidence interval are 0.917 and 0.997 , resp.), though included are a few outliers that are selected rather subjectively by an inspection. On the other hand, the relationship between the average SSN of an even-numbered cycle and the average SSN of the preceding odd-numbered cycle is very weak, for which the correlation coefficient is 0.537 whose lower and upper limits of the $95 \%$ confidence interval are -0.093 and 0.860 , respectively (Figure 7(b)). That is, the correlation is apparently observed in the even-odd pairs of cycles but is obscure in the odd-even pairs. The $P$ value in the null hypothesis test is $6.57^{-7}$ for the correlation of the even-odd pairs, while that for the odd-even pairs is 0.083 . Therefore, the former correlation is accepted at a very high significance level, but the latter correlation is rejected at the significance level of $5 \%$. 


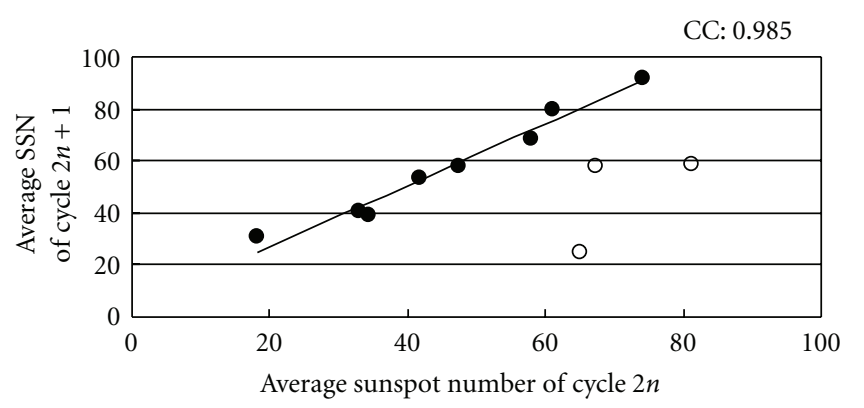

(a)

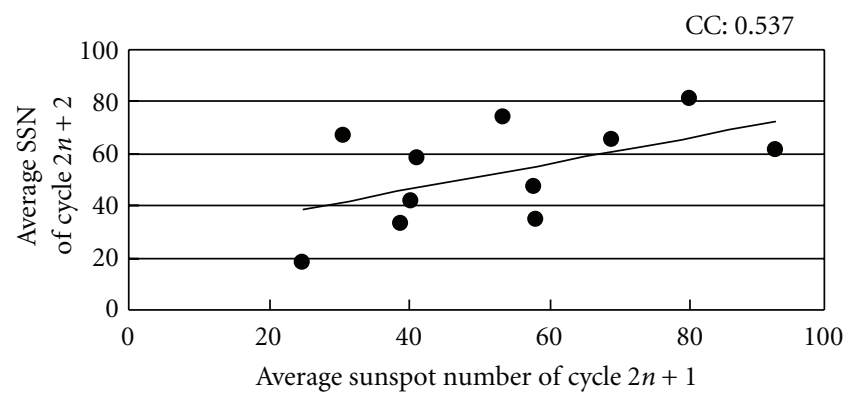

(b)

FIgURE 7: (a) Average sunspot number of odd-numbered solar cycles versus that of the preceding even-numbered cycles showing correlation between average SSN of solar cycle $2 n$ and that of solar cycle $(2 n+1)$, where $n$ represents an integer. (b) is the same as (a) but showing average sunspot number of even-numbered solar cycles $(2 n+2)$ versus that of the preceding odd-numbered cycles $(2 n+1)$.

We note here an interesting finding that may indicate intrinsic difference between even-numbered cycles and oddnumbered cycles. Figures 8 (a) and 8 (b) show relationships between the SSN at a point three years before the minimum and the average SSN (see Figure 4) for even-numbered cycles and for odd-numbered cycles, respectively. It is clear that the relationship seen in Figure $8(a)$ is much stronger. The correlation coefficient for even-numbered cycles is 0.956 $(0.833,0.989)$, while that for odd-numbered cycles is 0.738 $(0284,0.921)$, where numerals in parentheses show lower and upper limits of the $95 \%$ confidence interval. It is to be noted that no outliers that are observed in Figure 7(a) exist in Figure 8(a). The $P$ value in the null hypothesis test is $9.76^{-7}$ for the correlation between the SSN at a point three years before the minimum and the average SSN of the succeeding even cycle, while that for the correlation between the SSN at a point three years before the minimum and the average SSN of the succeeding odd cycle is 0.0048 .

A still more intriguing relationship, shown in Figure 9, indicates that the average of the SSN for $(2 n+1)$ cycles is related to those for $(2 n+4)$ cycles, where $n$ represents an integer. Although the correlation is not very strong that the correlation coefficient is $-0.697(-0.062,-0.930)$, the $P$ value in the null hypothesis test is 0.030 indicating that existence of the correlation cannot be rejected at the significance level of $5 \%$. We note here that the previously described intercycle relatedness shown in Figures $7(a)$ and 9 can be

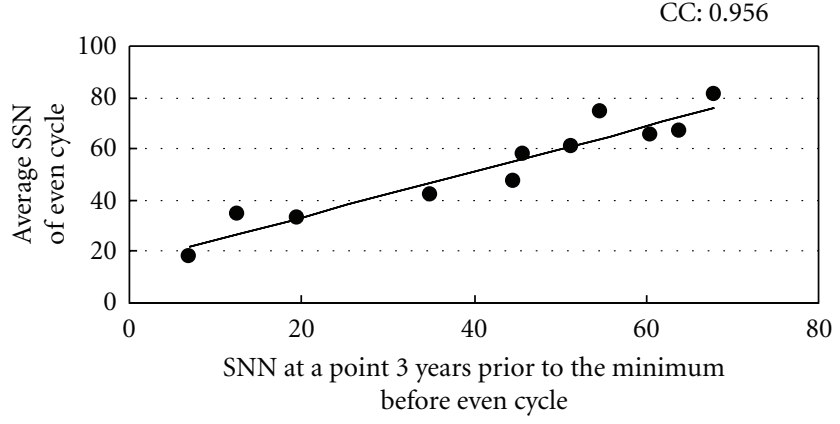

(a)

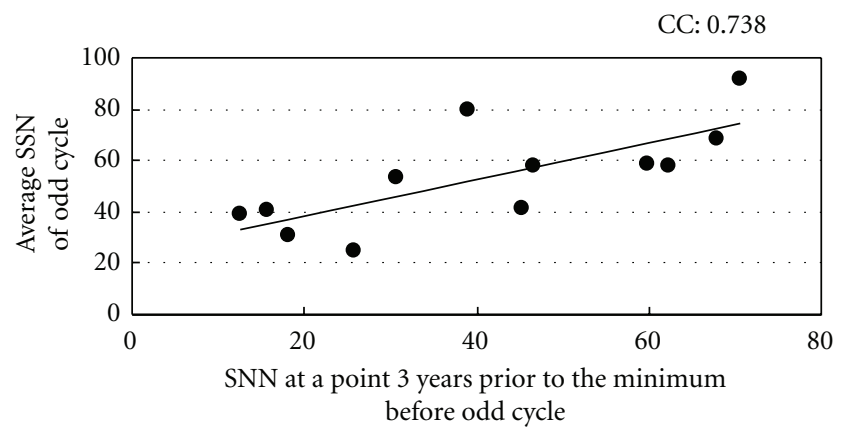

(b)

Figure 8: (a) Average sunspot number divided at a point three years before the minimum versus the sunspot number at the dividing point for even numbered cycles. (b) is the same as (a) but showing for odd numbered cycles.

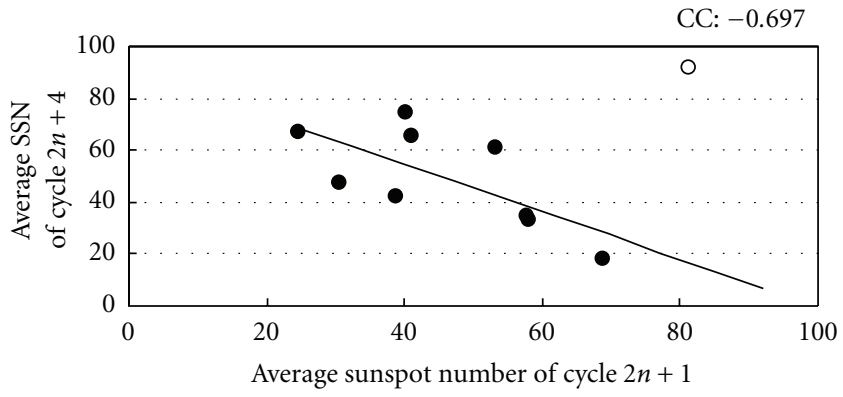

Figure 9: Scatter plot between the average sunspot number of odd-numbered cycles (abscissa) and that of the succeeding evennumbered cycle after the next cycle (ordinate), demonstrating an inverse relationship between the average sunspot number for $(2 n+$ $4)$ cycles and that for $(2 n+1)$ cycles. An outlier indicated by a white circle is omitted when the least-regression line is drawn.

seen when the maximum SSN is taken instead of the average SSN. However, the correlations are not as strong as when the average SSN is taken. We also add that no other significant intercycle relatedness besides those two shown in Figures 7(a) and 9 has been found.

When the positive correlation between the average SSN for $2 n$ cycles and that for $(2 n+1)$ cycles and the negative correlation between the average SSN for $(2 n+1)$ cycles and that for $(2 n+4)$ cycles are combined, it turns out that the cycles $1,4,5,8,9,12,13,16,17,20,21$, and 24 are interrelated 
on the one hand and the cycles $2,3,6,7,10,11,14,15,18$, 19,22 , and 23 reveal interrelatedness on the other. These two series of cycles do not mix or merge but, rather, constitute two independent series.

\section{Discussion}

The tendency of discreteness of the amplitude of solar cycle is most astonishing. The fact that the SSNs are likely to swarm near particular values around the peak of the solar cycle indicates that the tendency is not an artifact produced by the formula to calculate the SSN. Although we cannot explain the physical meaning of this remarkable finding at present, we think that our result imposes an important and serious constraint on future theoretical studies.

Another notable finding is the intercycle relatedness of the amplitude of solar cycles in which cycles are separated into two series that never mix nor merge. This remarkable result is derived from the positive correlation between the average SSN for even cycles and that for the succeeding odd cycles and the negative correlation between the average SSN for $(2 n+1)$ cycles and that for $(2 n+4)$ cycles. A few outliers observed in these correlations may not be due to the poor quality of early SSN. In fact, one of the outliers in the correlation between the average SSN of even cycles and that for the succeeding odd cycles is a pair at cycle 22 and cycle 23 (two other pairs are cycles 4-5 and cycles 8-9). At present, we do not have any idea about the appearance of outliers in the correlation. However, we would like to emphasize here that. if those outlier pairs are excepted, the amplitude of odd cycles tends to be larger than that of the preceding even cycles (see also Figure 1) and the ratio between the amplitudes is nearly the same (around 1.2).

We showed in this paper that the correlation between the maximum SSN and the average SSN as well as the correlation between the average SSN of the next cycle and the SSN at the dividing point becomes the best when solar cycles are divided at a point three years before the minimum. Why three years before the minimum?

It has been pointed out that the length of a solar cycle is correlated with the maximum SSN in the succeeding cycle which affords us an empirical means to predict the amplitude of the next solar cycle [2, 3]. Yoshida and Yamagishi [1] disclosed that what is actually essential in the correlation is the time for the SSN to fall from a value of 50 to its minimum value, not the whole length of the period; that is, the really significant parameter for the predictability is the decreasing rate of the SSN in the last several years before the minimum.

On the other hand, it has long been known empirically that the magnitude of a global geomagnetic index during the declining phase of solar activity serves as a useful predictor of the succeeding solar cycle amplitude [4-7]. Further, it has been discovered that the amplitude of the succeeding solar cycle can be predicted based on (1) the polar magnetic field on the sun during the three years prior to the minimum SSN [8-10] and (2) the azimuthally averaged radial magnetic field at the surface of the sun in the late stages of a solar cycle [11]. All these observations suggest that something significant which is indicative of the amplitude of the next cycle comes out on the surface of the sun in the final stage of a solar cycle.

We shortly comment here that the intensity of the interplanetary magnetic field which is directly related to the geomagnetic activity decreased parallel with the SSN in the last three years of solar cycle 23 [1]. Based on the finding they argued that the SSN in the last stage of the solar cycle is a good proxy of the poloidal magnetic field in the solar corona that should be the seed of the toroidal field of the sun in the next solar cycle and that the physical ground of the empirically well-known relationship between the minimum geomagnetic activity and the amplitude of the next solar cycle (e.g., [4]) might be given by the supposition.

A simple and reasonable way to explain both these observations as well as our finding that the correlation between the average SSN over a cycle and the SSN at a point three years before the minimum is strongest is the hypothesis that two cycles in the SSN overlap temporally with a phase lag as suggested by Cameron and Schüssler [11]. Actually, we see in the so-called butterfly diagram that high-latitude sunspots of the new cycle begin to appear when the old cycle is still in progression in low latitudes, and we have known that stronger cycles rise more quickly toward their sunspot maximum [12]. Cameron and Schüssler [11], demonstrating that when the following cycle is strong, the point at the minimum SSN actually moves to an earlier time making the length of the preceding cycle shorter, suggested that the faster rise of a stronger following cycle which overlaps with the preceding cycle leads to an earlier sunspot minimum than in the case of a weaker follower. The observational fact that the maximum SSN is well correlated with the increasing rate at the rising phase of the cycle [13] would seem to support their postulation.

\section{Summary}

We have shown that the correlations between the average SSN and the maximum SSN of the cycle as well as between the SSN at the dividing point of a solar cycle and the average of the SSN over the following cycle are strongest when the dividing point is set at three years before the minimum SSN. Following is our remarkable findings when the average of the SSN is taken as representative of the solar amplitude.

(1) The values of the average of the SSN show a tendency of discreteness to fall around multiples of 20 . The tendency is supported by the observation that SSNs near the solar maximum are likely to take around some particular values.

(2) A positive relationship is seen between the average of the SSN of even-numbered cycles and that of the succeeding odd-numbered cycles, but the relationship is obscure between the pair of odd-numbered cycles and the succeeding even numbered cycles.

(3) On the other hand, an inverse relationship is seen between the average of the SSN for $(2 n+1)$ cycles and that for $(2 n+4)$ cycles.

The aforementioned two relationships separate solar cycles into two mutually independent series. 


\section{Acknowledgments}

The authors thank the SIDC-team, World Data Center for the Sunspot Index, Royal Observatory of Belgium, for the smoothed monthly mean SSNs. Critical reading by J. P. Rozelot and A. Kilcik improved the paper.

\section{References}

[1] A. Yoshida and H. Yamagishi, "Predicting amplitude of solar cycle 24 based on a new precursor method," Annales Geophysicae, vol. 28, no. 2, pp. 417-425, 2010.

[2] S. Watari, "Forecasting solar cycle 24 using the relationship between cycle length and maximum sunspot number," Space Weather, vol. 6, no. 12, Article ID S12003, 2008.

[3] D. H. Hathaway, R. M. Wilson, and E. J. Reichmann, "Group sunspot numbers: sunspot cycle characteristics," Solar Physics, vol. 211, no. 1-2, pp. 357-370, 2002.

[4] A. I. Ohl, "Forecast of sunspot maximum number of cycle 20," Solice Donie, vol. 9, p. 84, 1966.

[5] D. H. Hathaway and R. M. Wilson, "Geomagnetic activity indicates large amplitude for sunspot cycle 24," Geophysical Research Letters, vol. 33, no. 18, Article ID L18101, 2006.

[6] R. P. Kane, "A preliminary estimate of the size of the coming solar cycle 24, based on Ohl's precursor method," Solar Physics, vol. 243, no. 2, pp. 205-217, 2007.

[7] N. J. Bhatt, R. Jain, and M. Aggarwal, "Prediction of the maximum amplitude and timing of sunspot cycle 24," Solar Physics, vol. 260, no. 1, pp. 225-232, 2009.

[8] K. H. Schatten, P. H. Scherrer, L. Svalgaard, and J. M. Wilcox, "Using dynamotheory to predict the sunspot number during solar cycle 21," Geophysical Research Letters, vol. 5, p. 411, 1978.

[9] K. Schatten, "Fair space weather for solar cycle 24," Geophysical Research Letters, vol. 32, no. 21, Article ID L21106, pp. 1-4, 2005.

[10] L. Svalgaard, E. W. Cliver, and Y. Kamide, "Sunspot cycle 24: smallest cycle in 100 years?" Geophysical Research Letters, vol. 32, no. 1, pp. 1-4, 2005.

[11] R. Cameron and M. Schüssler, "Solar cycle prediction using precursors and flux transport models," Astrophysical Journal, vol. 659, no. 1, pp. 801-811, 2007.

[12] M. Waldmeier, "Neue eigenshaften der sonnenfleckenkurve," Astronomische Mitteilungen Zürich, vol. 14, pp. 105-130, 1935.

[13] R. J. Thompson, "The rise of solar Cycle number 22," Solar Physics, vol. 117, no. 2, pp. 279-289, 1988. 

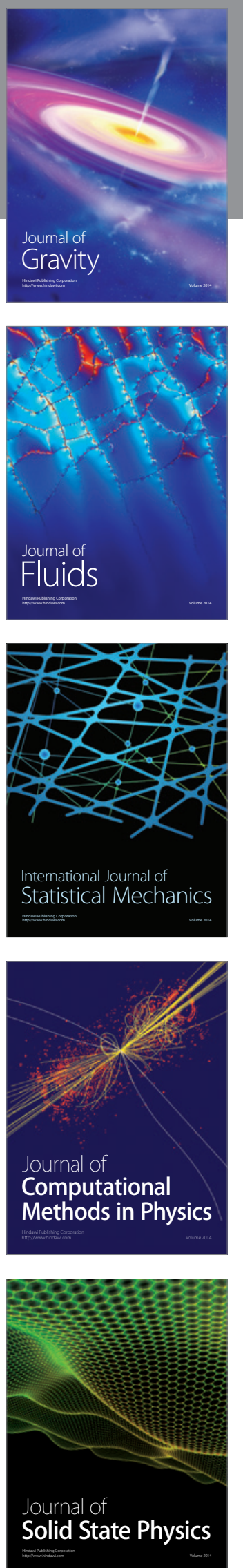

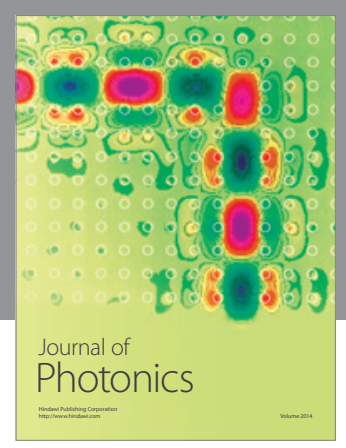

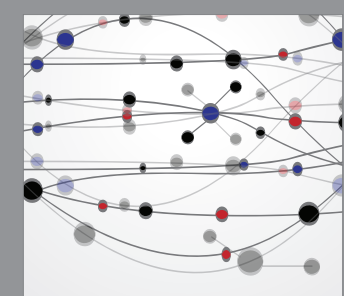

The Scientific World Journal
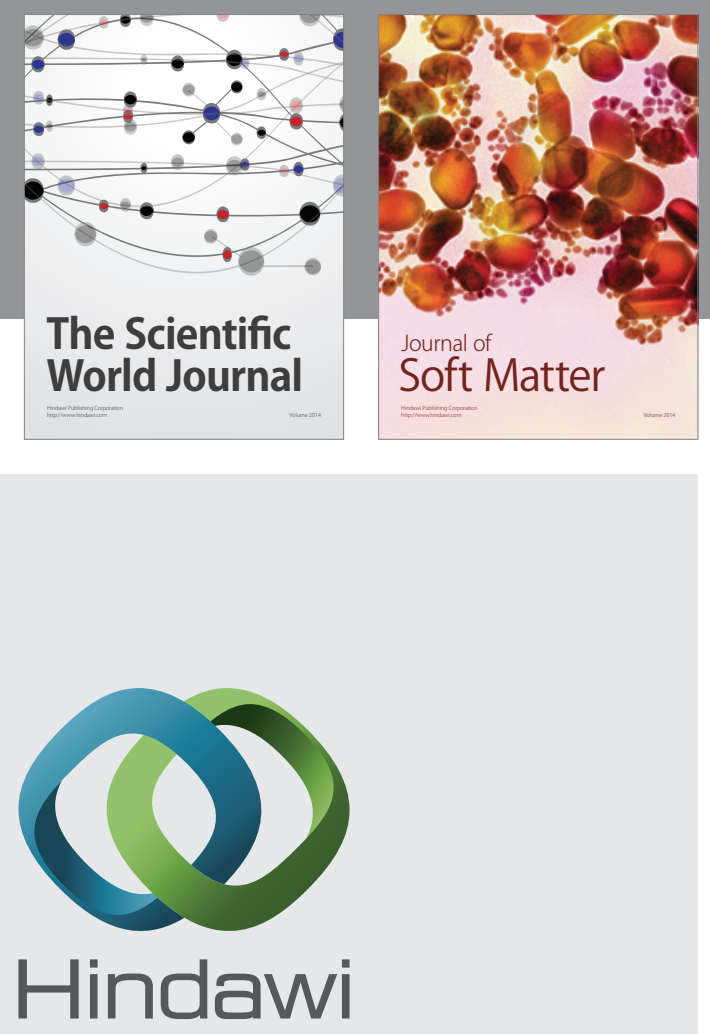

Submit your manuscripts at

http://www.hindawi.com
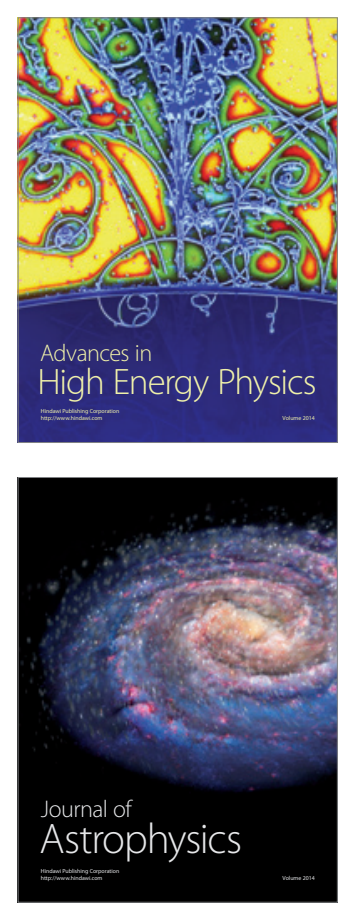
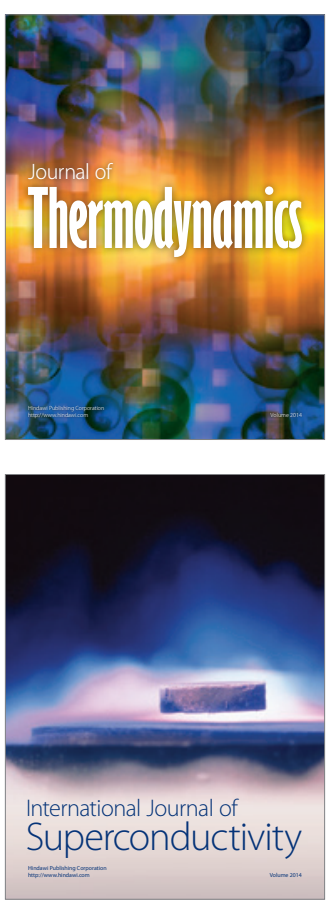
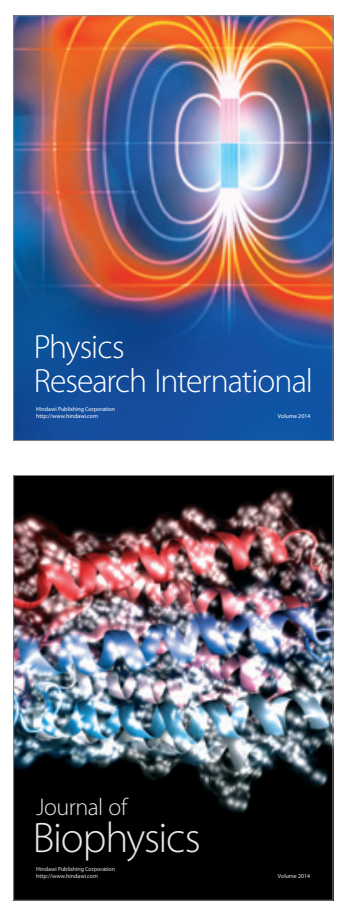
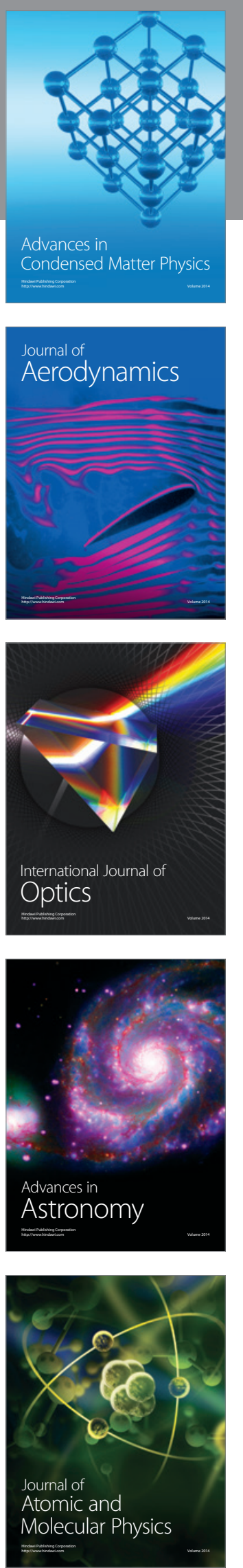